\title{
Online Gain Switching Algorithm for Joint Position Control of a Hydraulic Humanoid Robot
}

\author{
Jung-Yup Kim ${ }^{*}$, Christopher G. Atkeson ${ }^{*}$, Jessica K. Hodgins ${ }^{*}$, Darrin C. Bentivegna ${ }^{* * *}$ and Sung Ju Cho \\ ${ }^{*}$ Robotics Institute, Carnegie Mellon University, Pittsburgh, PA 15213, USA \\ ${ }^{* *}$ ATR Computational Neuroscience Laboratories, Dept. of Humanoid Robotics and Computational Neuroscience, Kyoto, JAPAN
}

\{jungyup, cga and jkh\}@cs.cmu.edu , darrin@\{cmu.edu or atr.jp\}, sunjuc@andrew.cmu.edu

\begin{abstract}
This paper proposes a gain switching algorithm for joint position control of a hydraulic humanoid robot. Accurate position control of the lower body is a one of the basic requirements for robust balance and walking control. In general, it is more difficult to perform joint position control of hydraulic humanoid robots than electric humanoid robots because of a slower actuator time constant and the backdrivability of hydraulic joints. Because of the backdrivability, external forces and torques have a large effect on the position of the joints, and external ground reaction forces prevent a simple PD joint controller from realizing accurate joint position control. We propose a state feedback controller for joint position control of the lower body, define three modes of state feedback gains, and switch the gains effectively according to the Zero Moment Point (ZMP) using linear interpolation. The performance of the algorithm is evaluated with a dynamic simulation of a hydraulic humanoid.
\end{abstract}

Index Terms - Gain switching, Hydraulic humanoid, Joint position control, $\mathrm{ZMP}$

\section{INTRODUCTION}

At present, most human-sized biped humanoid robots are powered by electric motors [1-3]. As small electric motors produce relatively small torques, they are generally used together with reduction gears to increase the torque. There are a number of advantages to this design: the hardware is simple and compact, the torque can be easily increased with reduction gears, there is no angular position limit, and accurate joint position control can be realized because the reduction gear prevents the transfer of external torques through the electric motor. Therefore, electric robots have good robustness in position control. However, force control is difficult because of the stiffness created by the reduction gear. The electric motor has a velocity limit, so the maximum torque is limited as well. In particular, the backlashless harmonic reduction gears that are commonly used in humanoid robots have lower torque limits than general planetary reduction gears. Therefore, electric motors and reduction gears may not be suitable for an adult-sized biped humanoid robot that must be robust to disturbances and uneven terrain.

An alternative approach is to use hydraulics for biped humanoid robots [4-8]. Hydraulic actuators for biped humanoid robots can produce a large torque for their size, have efficient power distribution, easy force control and backdrivability, and have a low rotational inertia due to the absence of a reduction gear. However, the hydraulics requires an oil compressor, making a self-contained system difficult, and oil lines generate external disturbances. In addition, the rotational ranges of the joints are limited due to the linkages required for rotary actuators. Most importantly, position control is difficult because of backdrivable joints and the large ground reaction forces seen during walking.

We propose an online gain switching algorithm for accurate position control of a hydraulic humanoid robot. Some researchers have studied online gain switching of robot manipulators for robust and accurate position control $[9,10]$, but online gain switching for biped robots has not yet been explored. As a first step, we applied the algorithm to a simulation of the lower body of the Sarcos Primus System (Fig. 1) because the control performance of the lower body is directly related to biped walking. Joint dynamic models were derived from frequency response testing and a simple mass damper model. We then designed a state feedback controller based on a Linear Quadratic Regulator (LQR) as a joint position controller. Three gain modes were chosen, and the gains are changed online according to the location of the ZMP and linear interpolation. The algorithm was verified with a dynamic simulation. All joints of the lower body could track desired trajectories with good accuracy.
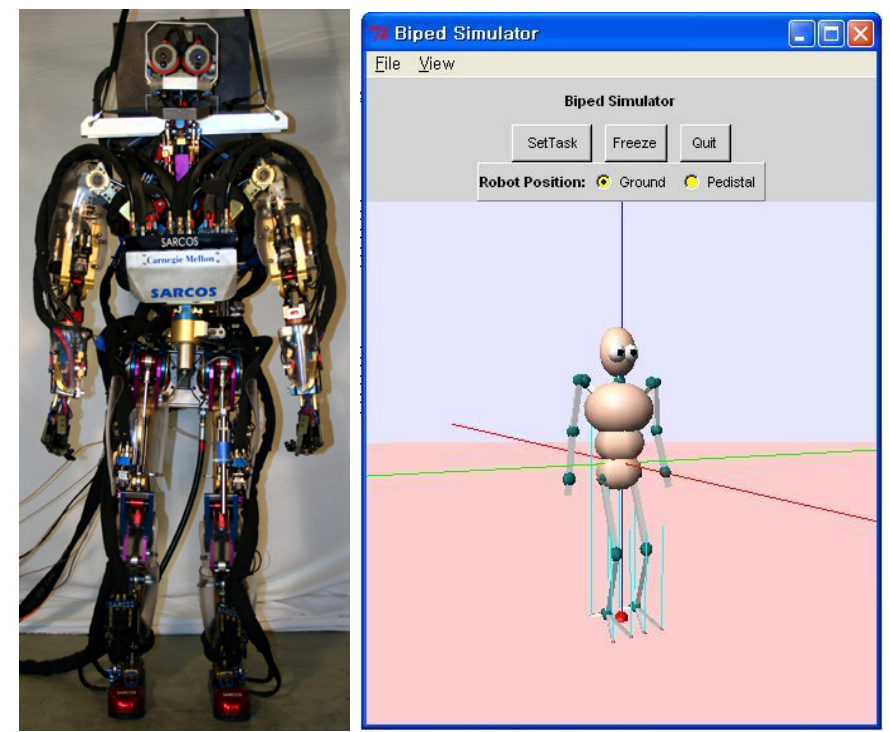

Fig. 1 Sarcos Primus System and simulator [11] 


\section{HYDRAULIC BIPED HUMANOID}

The target robot is a hydraulic humanoid robot developed by Sarcos (Fig. 1). It is an adult-sized humanoid robot whose height and weight are $1.65 \mathrm{~m}$ and $80 \mathrm{~kg}$. There are 53 degrees of freedom including the two passive toe joints. All actuators are hydraulic except for the eyes (electric) and fingers (pneumatic). The control architecture is distributed with communication occurring via Ethernet. The main computer communicates with the sub-controllers at $1 \mathrm{kHz}$. Table I shows the specifications and dimensions of the Sarcos humanoid robot.

TABLE I

SPECIFICATION AND DimENSIONS OF THE SARCOS HUMANOID ROBOT

\begin{tabular}{|c|c|c|c|c|}
\hline \multirow{6}{*}{ DOF } & Eye & 2 & Neck & 3 \\
\hline & Mouth & 1 & Shoulder & 3 \\
\hline & Elbow & 1 & Wrist & 3 \\
\hline & Hand & 6 & Waist & 3 \\
\hline & Hip & 3 & Knee & 1 \\
\hline & Ankle & 3 & Toe & 1 (passive) \\
\hline Weight & \multicolumn{4}{|c|}{$80 \mathrm{~kg}$ including hydraulic lines } \\
\hline Sensors & \multicolumn{4}{|c|}{$\begin{array}{l}\text { Potentiometers and force sensors at all hydraulic joints, two 6- } \\
\text { axis force/torque sensors on the soles of the feet, two IMUs in } \\
\text { the head and body, and a stereo camera }\end{array}$} \\
\hline Control rate & \multicolumn{4}{|c|}{$1 \mathrm{kHz}$ main control rate, $5 \mathrm{kHz}$ local control rate } \\
\hline $\begin{array}{l}\text { Hydraulic } \\
\text { actuator }\end{array}$ & \multicolumn{4}{|c|}{ Max 3000 psi, flow control servovalve } \\
\hline \multicolumn{5}{|c|}{ Dimensions (m) } \\
\hline \multicolumn{2}{|c|}{ Upper leg } & 0.38174 & Shoulder to shoulder & 0.39145 \\
\hline \multirow{2}{*}{\multicolumn{2}{|c|}{$\begin{array}{l}\text { Lower leg } \\
\text { Foot size }\end{array}$}} & 0.38075 & Upper arm & 0.25771 \\
\hline & & $0.3 \times 0.1$ & Lower arm & 0.24082 \\
\hline \multicolumn{2}{|c|}{ Hip to hip } & \multirow{2}{*}{$\frac{0.17780}{0.59365}$} & Eye to eye & 0.07188 \\
\hline \multicolumn{2}{|c|}{ Hip to neck } & & Neck to eye & 0.13622 \\
\hline
\end{tabular}

\section{JoINT POSITION CONTROL STRATEGY}

Position control can either be independent for each joint or simultaneously designed for multiple joints. Rigid body dynamics and control design are easy for single joint controllers but motion of the other joints must be treated as disturbances. Multiple joint designs require more complicated rigid body dynamics and controller design, but are more robust to disturbances. In this paper, we designed single joint controllers as an initial step toward a robust lower body controllers.

\section{A. Linear State Space Model of Joints}

To design a state feedback controller, we need a linear state space model of each joint. However, it is hard to derive exact joint models because many joints are connected in series, and the motion of one affects the others. We derived joint models by using a simple theoretical model and several experimental results.

The linear state space model of the left hip pitch joint was derived by a frequency response experiment:

$$
\dot{x}=A x+B u, \quad x=\left[\begin{array}{lll}
\theta & \dot{\theta} & \tau
\end{array}\right]^{T}
$$

In equation (1), $\quad A=\left[\begin{array}{ccc}0 & 1 & 0 \\ -14.2 & -0.98 & 0.22 \\ 0 & -4441.3 & -14.14\end{array}\right], B=\left[\begin{array}{c}0 \\ 0 \\ 1.97\end{array}\right]$

The state variables are angular position $\theta$ (rad), angular velocity $\dot{\theta}(\mathrm{rad} / \mathrm{sec})$ and joint torque $\tau(\mathrm{Nm})$. The input is a valve command $(-32000 \sim 32000, \mathrm{D} / \mathrm{A}$ units $)$ to the flow control servo valve. We fixed the pelvis center of the robot on a stand, both legs were in the air, and the other joints maintained angular positions using PD control or they were held against a mechanical stop. Fig. 2 shows a comparison of Bode plots of the experimental data and the model. The input is the valve command and the output is the joint torque.

The second row of $A$ represents the rigid body dynamics and the third rows of $A$ and $B$ represent the hydraulic actuator dynamics. The rigid body dynamics vary according to the mass distribution, hence each joint has a different $A$ matrix. The hydraulic actuator dynamics change based on the actuator specification. Therefore, we assumed each joint model is a mass-damper model as shown in Fig. 3, and derived the second row of $A$ using 3D CAD data assuming all other joints were fixed in a standard posture.
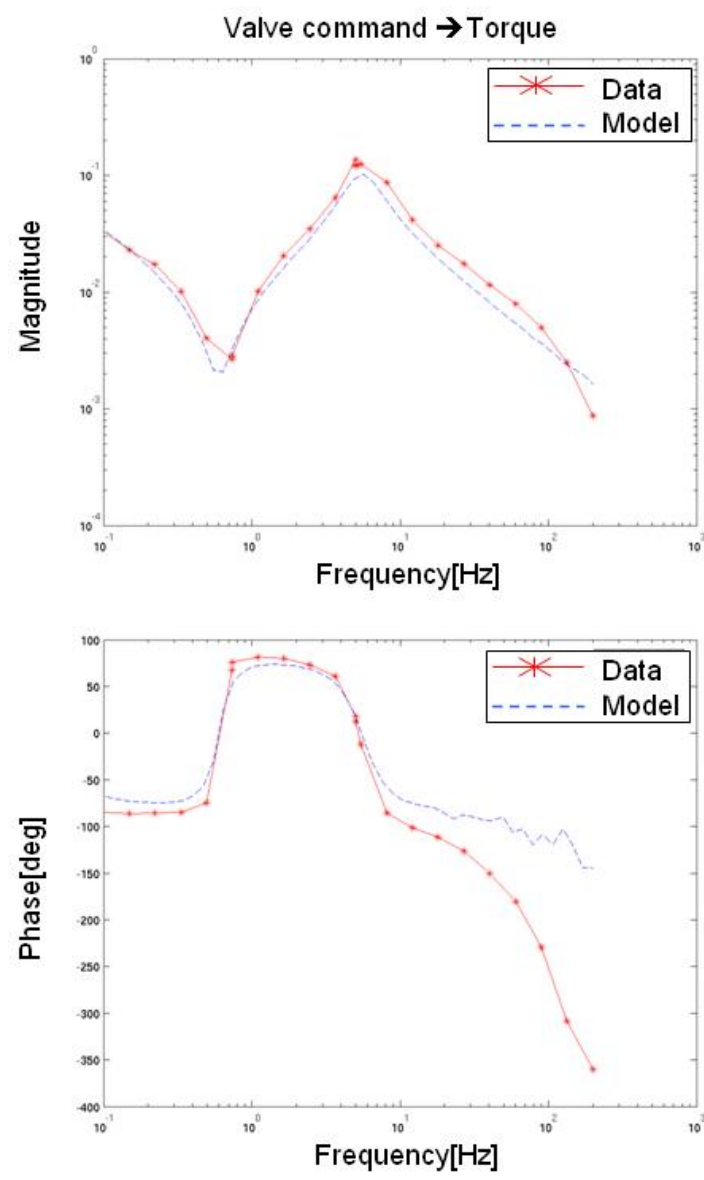

Fig. 2 Comparison of the Bode plot of the experimental data and the model .(Input : valve command, Output : joint torque) 


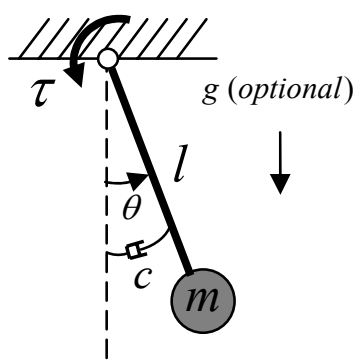

Fig. 3 Mass-damper model

In addition, we assume that the damping coefficient, $c$ of the joint model is same for all joints. The $c$ was calculated as 4.45 (Nmsec/rad) by comparing the experimental $A$ in equation (1) and the theoretical $A$ as shown in equation (2).

$$
A=\left[\begin{array}{ccc}
0 & 1 & 0 \\
-\frac{g}{l} & -\frac{c}{m l^{2}} & \frac{1}{m l^{2}} \\
0 & -4441.3 & -14.14
\end{array}\right]
$$

We derived the hydraulic actuator dynamics using frequency response experiments for the ankle joints and for the other leg joints. The form of the model was $\dot{\tau}=a \dot{\theta}+b \tau+c u$. The first term represents a rate of change of torque due to piston linear velocity, the second term represents a rate of change of torque due to piston leaks, and the third term represents a rate of change of torque due to the valve opening. The coefficients of the model should be functions of the joint angle as shown: $\dot{\tau}=-C^{2}(\theta) a \dot{\theta}-b \tau+C(\theta) c u$ because the moment arm varies with the angle. However, in this paper, we assume that the moment arm is constant for simplicity. We computed following models for the joints of the Sarcos humanoid robot:

Ankle joints: $\dot{\tau}=-3078.9 \dot{\theta}-4.43 \tau+1.44 u$

Other joints: $\dot{\tau}=-4441.3 \dot{\theta}-14.14 \tau+1.97 u$

$$
\begin{aligned}
& \text { Hip pitch/roll joint : } A=\left[\begin{array}{ccc}
0 & 1 & 0 \\
-14.2 & -0.98 & 0.22 \\
0 & -4441.3 & -14.14
\end{array}\right] B=\left[\begin{array}{c}
0 \\
0 \\
1.97
\end{array}\right] \\
& \text { Hip yaw joint : } A=\left[\begin{array}{ccc}
0 & 1 & 0 \\
0 & -31.44 & 7.06 \\
0 & -4441.3 & -14.14
\end{array}\right] \quad B=\left[\begin{array}{c}
0 \\
0 \\
1.97
\end{array}\right] \\
& \text { Knee pitch joint : } A=\left[\begin{array}{cccc}
0 & 1 & 0 \\
-45.42 & -13.833 & 3.11 \\
0 & -4441.3 & -14.14
\end{array}\right] B=\left[\begin{array}{c}
0 \\
0 \\
1.97
\end{array}\right] \\
& \text { Ankle pitch/roll joint : } A=\left[\begin{array}{ccc}
0 & 1 & 0 \\
-98.1 & -218.65 & 49.14 \\
0 & -3078.9 & -4.43
\end{array}\right] \quad B=\left[\begin{array}{c}
0 \\
0 \\
1.44
\end{array}\right] \\
& \text { Ankle yaw joint : } A=\left[\begin{array}{ccc}
0 & 1 & 0 \\
0 & -64.54 & 14.5 \\
0 & -3078.9 & -4.43
\end{array}\right] \quad B=\left[\begin{array}{c}
0 \\
0 \\
1.44
\end{array}\right]
\end{aligned}
$$

\section{B. Design of State Feedback Controller}

We use the state feedback controller to perform joint position control for the lower body:

$$
\begin{aligned}
u & =-k_{1} \theta-k_{2} \dot{\theta}-k_{3} \tau+k_{f f} \theta_{\text {desired }} \\
& =k_{1}\left(\theta_{\text {desired }}-\theta\right)-k_{2} \dot{\theta}-k_{3} \tau+k_{4} \theta_{\text {desired }}
\end{aligned}
$$

where, $k_{1}, k_{2}$ and $k_{3}$ are the state feedback gains, and $k_{f f}$ $\left(=k_{1}+k_{4}\right)$ is the feedforward gain for the tracking control, $k_{4}$ compensates for steady state error, $\theta$ is the actual angle, $\dot{\theta}$ is the actual angular velocity, $\tau$ is the actual torque, and $\theta_{\text {desired }}$ is the desired angular position. To decide the state feedback gains, we used LQR first and then refined the gains by hand through simulation. Because a biped walking robot periodically has ground contact, we cannot expect good performance of joint position control at all times with constant gains. Therefore, three gain modes for each leg were defined according to the contact state of the feet. Fig. 4 shows the gain modes. The low gain mode represents the swing leg in the single support phase. In this case, all proportional gains $\left(k_{l}\right)$ of the swing leg should low because there is no load. The high gain mode represents the supporting leg in single support phase. In this phase, the largest dynamic load that may be more than the robot's weight is applied to the foot and therefore, the proportional gains of this mode should be the largest among the three modes. The middle gain mode represents the supporting leg in the double support phase. In this case, the dynamic load is lower than the robot's weight but not zero. The proportional gains should be between the two other modes.

The gains of all joints of the leg in the low gain mode were determined by defining suitable $\mathrm{Q}$ and $\mathrm{R}$ matrices for $\mathrm{LQR}$, and then tuned by a simulation of tracking control. The initial values for $\mathrm{Q}$ and $\mathrm{R}$ for all joints were

$$
Q=\left[\begin{array}{ccc}
10^{10} & 0 & 0 \\
0 & 10^{6} & 0 \\
0 & 0 & 10^{4}
\end{array}\right] \text { and } \mathrm{R}=10^{-1}
$$

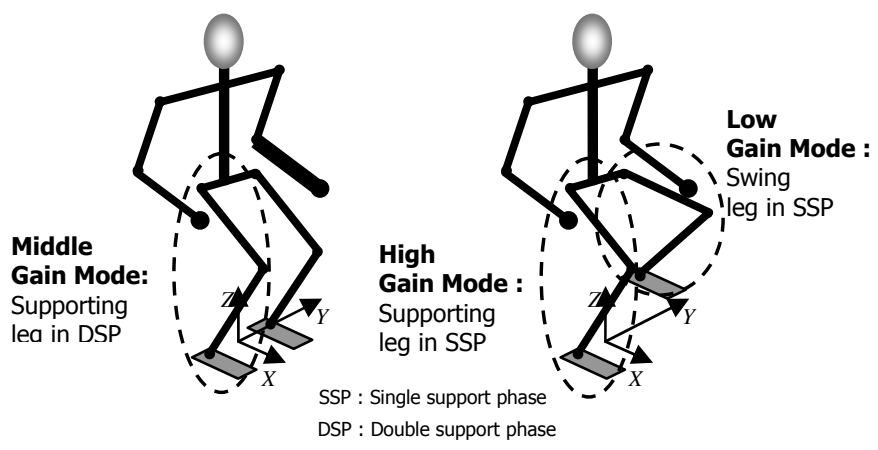

Fig. 4 Three gain modes 
After the gains of the low gain mode were decided for all joints, we then adjusted them to provide good control in double and single support. While simulating up-down, and side to side motions, we increased $k_{l}$ until the tracking error is small. If vibrations occur, we increased $k_{2}$. If the rate of change of the torque is high, we increased the $k_{3}$. Finally, $k_{f f}$ was tuned by measuring the steady state tracking error. In this manner, we could use simulated experiments to set the gains of the three modes. Table II shows the gains of the right knee pitch joint.

TABLE II

Gains of Right KneE Pitch Joint in EACH Mode

\begin{tabular}{|l|c|c|c|c|}
\hline & $k_{1}$ & $k_{2}$ & $k_{3}$ & $k_{4}$ \\
\hline Low Gain & 540840.897 & 1882.937 & 443.566 & -5408.409 \\
\hline Middle Gain & 811261.346 & 1882.937 & 221.783 & -8112.614 \\
\hline High Gain & 1622522.69 & 1882.937 & 177.4264 & -16225.227 \\
\hline
\end{tabular}

\section{Online Gain Switching Algorithm}

We designed a suitable algorithm to smoothly switch between gain modes. We use ZMP, which is equal to the center of the ground reaction force on the ground, to calculate the dynamic load distribution between the legs. Fig. 5 shows the three gain switching boundaries. $D$ is the lateral distance between the origin of the ZMP coordinate frame and the ankle joint. The ZMP coordinate frame is placed on the center position between the two projected soles on the ground. The boundaries 1 and 3 are placed $0.8 D$ to the right and left from the origin, and the boundary 2 is placed on the origin. Fig. 6 shows the schematic of the online gain switching algorithm. If the $Y_{Z M P}$ is to the right of boundary 3, the right leg is in the low gain mode and the left leg is in the high gain mode. If the $Y_{Z M P}$ is to the left of boundary 1, the right leg is in the high gain mode, and the left leg is in the low gain mode. If the $Y_{Z M P}$ is on boundary 2, both legs are in the middle gain mode. In the areas near boundaries, the gains linearly interpolated to create a smooth transition.

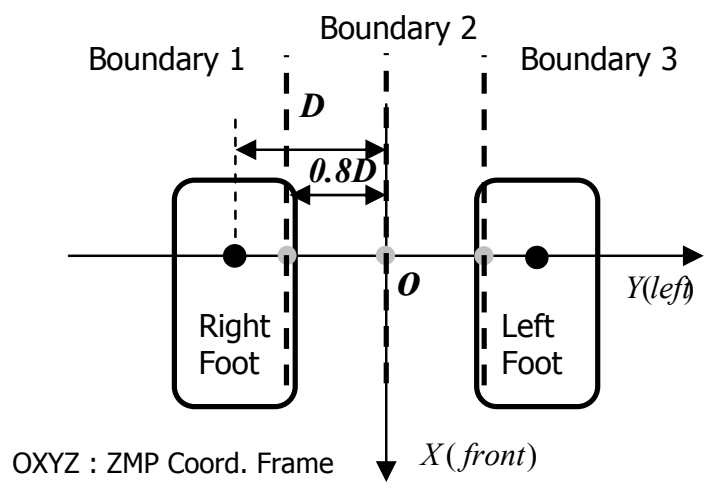

Fig. 5 Gain switching boundaries



Fig. 6 Schematic of online gain switching algorithm

\section{SimUlation}

\section{A. Simulator}

We performed dynamic simulations to test the performance of the proposed gain switching algorithm. The SL simulation and Real-Time Control Software Package was used for a simulator as shown in Fig. 1 [11]. We used 3D CAD data to create a simulator that matched the physical humanoid shown in Fig. 1. The ground contact model is a damped-spring static friction model. The hydraulic actuator model was implemented for all joints of the lower body and the state feedback controllers designed in Section III were used. For the upper body, simple PD servo controllers were used.

\section{B. Joint Position Control Test in the Air}

Joint position control in the air was performed to test the low gain mode in simulation. The pelvis center was fixed in 3D space. A prescribed walking gait at $1 \mathrm{sec} / \mathrm{step}$ and $0.25 \mathrm{~m}$ step length was used to generate desired joint trajectories. Fig. 7 and Table III show the desired and actual joint trajectories of the right leg and the maximum and average tracking errors. The errors in the plots were magnified by a factor of 10 for visualization. The average errors are less than $0.0026 \mathrm{rad}$. The maximum error in all joints is $0.0101 \mathrm{rad}$ for the left hip pitch joint. However, the average error is only $0.0025 \mathrm{rad}$. These results show that this control scheme provided good position control.

\section{Joint Position Control Test on the Ground}

The joint position control in double support phase was used to test the gains and the switching algorithm. Up-down motion was used to test the middle gains only and side to side motion was used to test the three gain modes and switching between them. The peak to peak amplitudes are $0.08 \mathrm{~m}$ and $0.12 \mathrm{~m}$ respectively, and the frequencies are $0.5 \mathrm{~Hz}$ for up/down and side to side motions. These values were determined by approximating the magnitudes and frequencies seen in human walking. Fig. 8 shows the desired and actual angles of the several joints during the up-down motion. The largest maximum error was $0.0069 \mathrm{rad}$ at the knee pitch joint. The average errors were less than $0.0036 \mathrm{rad}$. This result shows 
that the middle gains are decided well. Next, we performed side to side motions with or without the switching algorithm. Without the switching algorithm, the simulation fell down due to the poor tracking performance. The maximum tracking error was $0.0446 \mathrm{rad}$ of the left hip roll joint, and the average errors were about $0.0050 \mathrm{rad}$. With the switching algorithm, the simulator performed the side to side motion well. Fig. 9 represents the desired and actual angles of all joints and $Y_{Z M P}$. Even though $Y_{Z M P}$ moves quickly by $0.1 \mathrm{~m}$, the tracking performance is good. The maximum tracking error and average error were only $0.0069 \mathrm{rad}$ and $0.00142 \mathrm{rad}$ respectively in Table IV. Therefore, all gains seem to be appropriate, and the proposed gain switching algorithm is effective for accurate position control, considering no jerk occurred and tracking errors were always small.
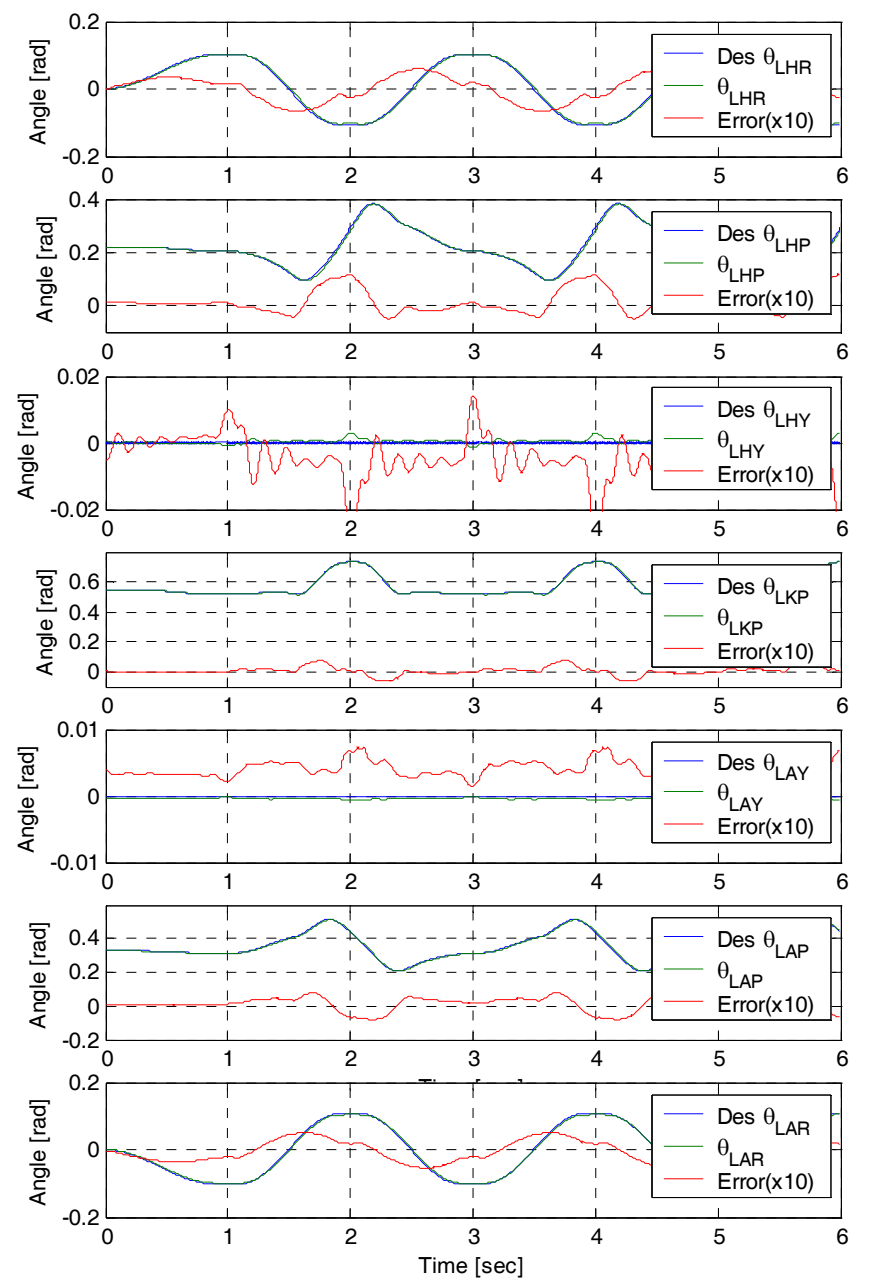

Fig. 7 Simulation result of joint position control in low gain mode
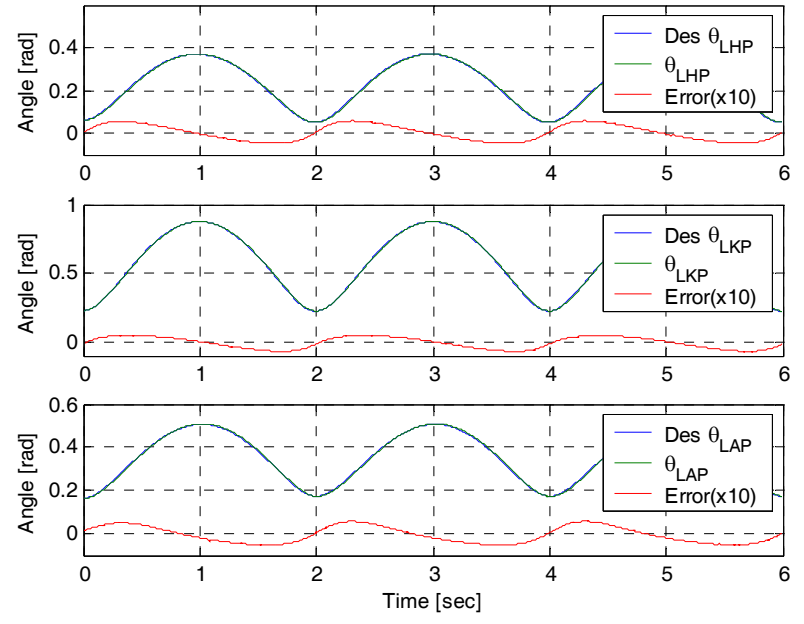

Fig. 8 Simulation result of joint position control during up-down motion
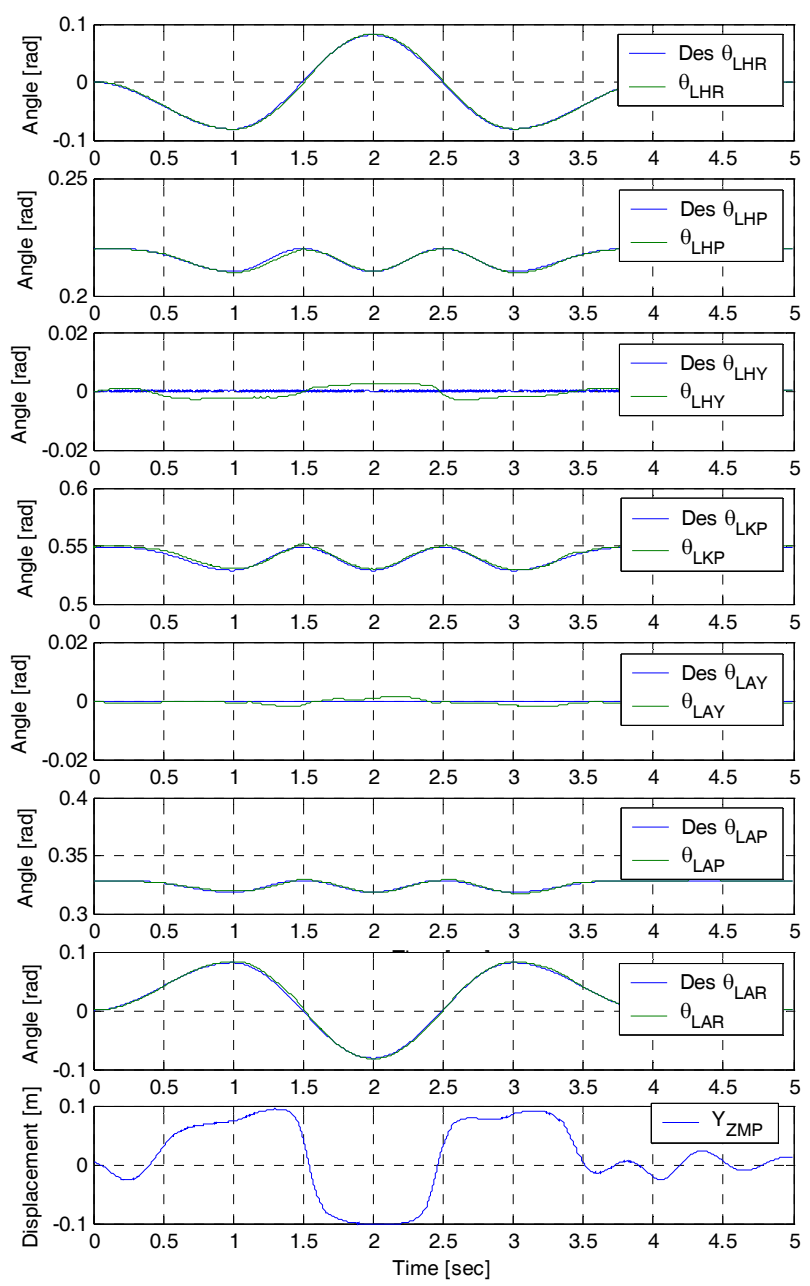

Fig. 9 Simulation result of joint position control during side to side motion 
TABLE III

The Maximum And Average Tracking ERRORS IN THE AIR

\begin{tabular}{|c|c|c|}
\hline Joint & Max. Tracking Error & Avg. Tracking Error \\
\hline Left Hip Roll(LHR) & $0.0061 \mathrm{rad}$ & $0.0026 \mathrm{rad}$ \\
\hline Left Hip Pitch(LHP) & $0.0101 \mathrm{rad}$ & $0.0025 \mathrm{rad}$ \\
\hline Left Hip Yaw(LHY) & $0.0031 \mathrm{rad}$ & $0.0006 \mathrm{rad}$ \\
\hline Left Knee Pitch(LKP) & $0.0081 \mathrm{rad}$ & $0.0014 \mathrm{rad}$ \\
\hline Left Ankle Yaw(LAY) & $0.0007 \mathrm{rad}$ & $0.0004 \mathrm{rad}$ \\
\hline Left Ankle Pitch(LAP) & $0.0056 \mathrm{rad}$ & $0.0023 \mathrm{rad}$ \\
\hline Left Ankle Roll(LAR) & $0.0050 \mathrm{rad}$ & $0.0023 \mathrm{rad}$ \\
\hline
\end{tabular}

TABLE IV

The Maximum And Average Tracking ERrors on THE Ground

\begin{tabular}{|c|c|c|}
\hline Joint & Max. Tracking Error & Avg. Tracking Error \\
\hline Left Hip Roll(LHR) & $0.0039 \mathrm{rad}$ & $0.0012 \mathrm{rad}$ \\
\hline Left Hip Pitch(LHP) & $0.0017 \mathrm{rad}$ & $0.0003 \mathrm{rad}$ \\
\hline Left Hip Yaw(LHY) & $0.0029 \mathrm{rad}$ & $0.0014 \mathrm{rad}$ \\
\hline Left Knee Pitch(LKP) & $0.0034 \mathrm{rad}$ & $0.0013 \mathrm{rad}$ \\
\hline Left Ankle Yaw(LAY) & $0.0021 \mathrm{rad}$ & $0.0008 \mathrm{rad}$ \\
\hline Left Ankle Pitch(LAP) & $0.0015 \mathrm{rad}$ & $0.0005 \mathrm{rad}$ \\
\hline Left Ankle Roll(LAR) & $0.0069 \mathrm{rad}$ & $0.0012 \mathrm{rad}$ \\
\hline
\end{tabular}

\section{ZMP Control in Double Support Phase (DSP)}

We implemented ZMP control to demonstrate ZMP based walking in simulation. The ZMP controller was designed by modeling the robot as a simple inverted pendulum with a flexible joint that has a spring constant. By using ZMP feedback, the state variables were tracked by a full state observer, and then a state feedback controller was designed, which uses a horizontal movement of the pelvis center as a control input. Fig. 10 shows the result of ZMP control in DSP. In the figure, the initial overshoots occur because the simple inverted pendulum model is a non-minimum phase system. This successful result of ZMP control in DSP demonstrates that the position control of the lower body is appropriate for biped walking control.

\section{CONCLUSION}

This paper proposed a gain switching algorithm for joint position control of a hydraulic humanoid robot. Our simulation experiments demonstrated that it is possible to obtain accurate joint position control by switching gains based on the location of the ZMP. We plan to apply the control scheme to the Sarcos hydraulic humanoid robot, and test the robot's performance with similar experiments. We will implement and test more complex controller designs that take varying moment arms and dynamics into account. We will also consider a controller design for multiple joints rather than independent joint control.

\section{ACKNOWLEDGMENT}

This material is based upon work supported in part by the National Science Foundation under grants CNS-0224419, ECS-0325383, and EEC-0540865.
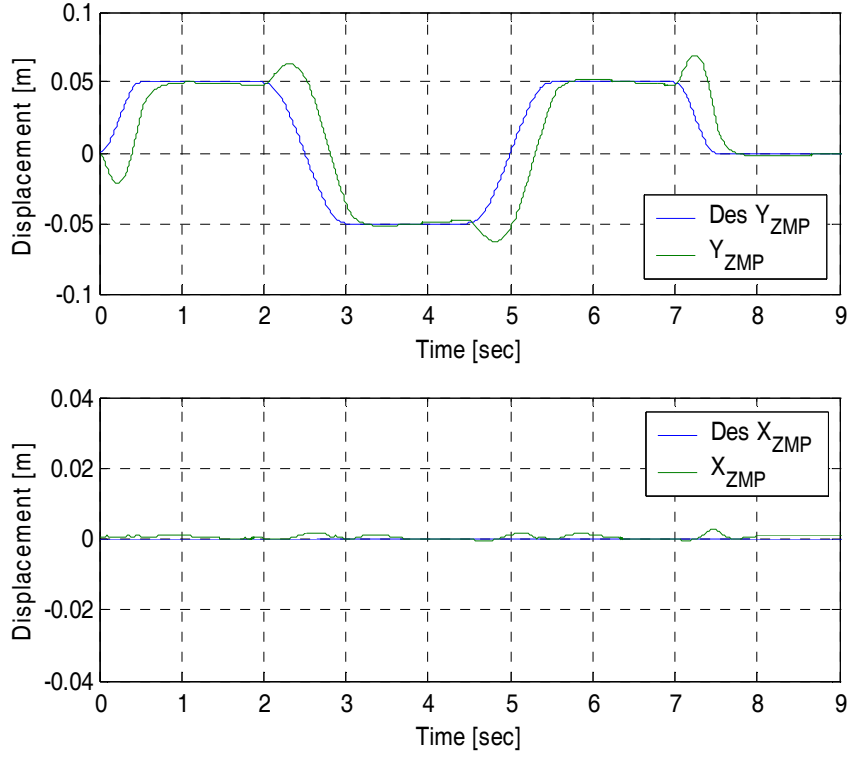

Fig. 10 ZMP control simulation in double support phase

\section{REFERENCES}

[1] Y. Sakagami, R. Watanabe, C. Aoyama, S. Matsunaga, N. Higaki, and K. Fujimura,"The intelligent ASIMO: System overview and integration," in Proc. IEEE/RSJ Int. Conf. on Intelligent Robots and Systems, pp. 24782483, 2002.

[2] K. Akachi, K. Kaneko, N. Kanehira, S. Ota, G. Miyamori, M. Hirata, S. Kajita, and F. Kanehiro, "Development of Humanoid Robot HRP-3P," in Proc. IEEE-RAS Int. Conf. on Humanoid Robotics, pp. 50-55, 2005.

[3] J. Y. Kim, I. W. Park, J. Lee, M. S. Kim, B. K. Cho, and J. H. Oh, "System Design and Dynamic Walking of Humanoid Robot KHR-2," in Proc. IEEE Int. Conf. on Robotics and Automation, pp. 1443-1448, 2005.

[4] S. O. Anderson, M. Wisse, C. G. Atkeson, J. K. Hodgins, G. J. Zeglin, and B. Moyer, "Powered Biped Based on Passive Dynamic Principles," in Proc. IEEE-RAS Int. Conf. on Humanoid Robotics, pp. 110-116, 2005.

[5] S. O. Anderson, C. G. Atkeson, and J. K. Hodgins, "Coordinating Feet in Bipedal Balance," in Proc. IEEE-RAS Int. Conf. on Humanoid Robotics, pp.1-5, 2006.

[6] S. H. Hyon, and Gordon Cheng, "Passivity-Based Full-Body Force Control for Humanoids and Application to Dynamic Balancing and Locomotion," in Proc. IEEE/RSJ Int. Conf. on Intelligent Robots and Systems, pp. 4915-4922, 2006.

[7] G. Cheng, S. H. Hyon, J. Morimoto, A. Ude, G. Colvin, and W. Scroggin, "CB: A Humanoid Research Platform for Exploring NeuroScience," in Proc. IEEE-RAS Int. Conf. on Humanoid Robotics, pp.182-187, 2006.

[8] S. H. Hyon, and G. Cheng, "Gravity Compensation and Full-Body Balancing for Humanoid Robots," in Proc. IEEE-RAS Int. Conf. on Humanoid Robotics, pp.214-221, 2006.

[9] M. A. Jarrah, and O. M. Al-Jarrah, "Position Control of a Robot Manipulation Using Continuous Gain Scheduling," in Proc. IEEE Int. Conf. on Robotics and Automation, pp. 170-175, 1999.

[10]B. Paijmans, W. Symens, H. V. Brussel, and J. Swevers, "GainScheduling Control for Mechanics Systems with Position Dependent Dynamics," in Proc. Int. Conf. on Noise and Vibration Engineering, 93105, 2006.

[11]S. Schaal, "The SL Simulation and Real-Time Control Software Package," unpublished. 